\title{
Short-term outcomes of extremely preterm infants at discharge: a multicenter study from Guangdong province during 2008- 2017
}

Fan Wu ${ }^{1,2+}$, Guosheng Liu ${ }^{1 *}$, Zhoushan Feng ${ }^{2+}$, Xiaohua Tan², Chuanzhong Yang ${ }^{3 \dagger}$, Xiaotong Ye ${ }^{3}$, Yiheng Dai ${ }^{4+}$, Weiyi Liang ${ }^{4}$, Xiuzhen $\mathrm{Ye}^{5 \dagger}$, Jing $\mathrm{Mo}^{5}$, Lu Ding ${ }^{6 \dagger}$, Benqing $\mathrm{Wu}^{6}$, Hongxiang Chen ${ }^{7 \dagger}$, Chiwang $\mathrm{Li}^{7}$, Zhe Zhang ${ }^{8 \dagger}$, Xiao Rong ${ }^{8}$, Wei Shen ${ }^{9 \dagger}$, Weimin Huang ${ }^{9}$, Bingyan Yang ${ }^{10 \dagger}$, Junfeng Lv $^{10}$, Leying Huo ${ }^{11 \dagger}$, Huiwen Huang ${ }^{11}$, Hongping Rao ${ }^{12 \dagger}$, Wenkang Yan ${ }^{12}$, Yong Yang ${ }^{13 \dagger}$, Xuejun Ren ${ }^{13}$, Fangfang Wang ${ }^{14 \dagger}$, Dong Liu ${ }^{14}$, Shiguang Diao ${ }^{15 \dagger}$, Xiaoyan Liu ${ }^{15}$, Qiong Meng ${ }^{16 \dagger}$, Yu Wang ${ }^{16}$, Bin Wang ${ }^{17 \dagger}$, Lijuan Zhang ${ }^{17}$, Yuge Huang ${ }^{18 \dagger}$, Dang Ao ${ }^{18}$, Weizhong $\mathrm{Li}^{19 \dagger}$, Jieling Chen ${ }^{19}$, Yanling Chen ${ }^{20 \dagger}$, Wei $\mathrm{Li}^{20}$, Zhifeng Chen ${ }^{21}$, Yueqin Ding ${ }^{21}$, Xiaoyu $\mathrm{Li}^{22 \dagger}$, Yuefang Huang ${ }^{22}$, Niyang $\mathrm{Lin}^{23+}$, Yangfan $\mathrm{Cai}^{23}$, Shasha Han ${ }^{1 \dagger}$, Ya Jin ${ }^{1}$, Zhonghe Wan ${ }^{24 \dagger}$, Yi Ban ${ }^{24}$, Bo Bai ${ }^{25+}$, Guanghong Li ${ }^{25}$, Yuexiu Yan ${ }^{26+}$ and Qiliang Cui ${ }^{2 *}$

\section{Abstract}

Background: An increasing number of extremely preterm (EP) infants have survived worldwide. However, few data have been reported from China. This study was designed to investigate the short-term outcomes of EP infants at discharge in Guangdong province.

Methods: A total of 2051 EP infants discharged from 26 neonatal intensive care units during 2008-2017 were enrolled. The data from 2008 to 2012 were collected retrospectively, and from 2013 to 2017 were collected prospectively. Their hospitalization records were reviewed.

(Continued on next page)

\footnotetext{
* Correspondence: tlgs@jnu.edu.cn; cuiqiliang55@126.com

${ }^{\dagger}$ Fan Wu, Zhoushan Feng, Chuanzhong Yang, Yiheng Dai, Xiuzhen Ye, Lu Ding, Hongxiang Chen, Zhe Zhang, Wei Shen, Bingyan Yang, Leying Huo, Hongping Rao, Yong Yang, Fangfang Wang, Shiguang Diao, Qiong Meng, Bin Wang, Yuge Huang, Weizhong Li, Yanling Chen, Zhifeng Chen, Xiaoyu Li, Niyang Lin, Shasha Han, Zhonghe Wan, Bo Bai and Yuexiu Yan contributed equally to this work.

'Department of Neonatology, the First Affiliated Hospital of Jinan University, Guangzhou 510630, Guangdong, China

${ }^{2}$ Department of Pediatrics, the Third Affiliated Hospital of Guangzhou

Medical University, Guangzhou 510150, Guangdong, China

Full list of author information is available at the end of the article
}

C) The Author(s). 2019 Open Access This article is distributed under the terms of the Creative Commons Attribution 4.0 International License (http://creativecommons.org/licenses/by/4.0/) which permits unrestricted use, distribution, and reproduction in any medium, provided you give appropriate credit to the original author(s) and the source, provide a link to the Creative Commons license, and indicate if changes were made. The Creative Commons Public Domain Dedication waiver (http://creativecommons.org/publicdomain/zero/1.0/) applies to the data made available in this article, unless otherwise stated. 
(Continued from previous page)

Results: During 2008-2017, the mean gestational age (GA) was $26.68 \pm 1.00$ weeks and the mean birth weight (BW) was $935 \pm 179 \mathrm{~g}$. The overall survival rate at discharge was 52.5\%. There were $321 \mathrm{infants}(15.7 \%)$ died despite active treatment, and 654 infants (31.9\%) died after medical care withdrawal. The survival rates increased with advancing GA and BW $(p<0.001)$. The annual survival rate improved from 36.2\% in 2008 to 59.3\% in 2017 ( $p<0.001)$. EP infants discharged from hospitals in Guangzhou and Shenzhen cities had a higher survival rate than in others $(p<0.001)$. The survival rate of EP infants discharged from general hospitals was lower than in specialist hospitals $(p<0.001)$. The major complications were neonatal respiratory distress syndrome, 88.0\% (1804 of 2051), bronchopulmonary dysplasia, 32.3\% (374 of 1158), retinopathy of prematurity (any grade), 45.1\% (504 of 1117), necrotizing enterocolitis (any stage), 10.1\% (160 of 1588), intraventricular hemorrhages (any grade), 37.4\% (535 of 1431), and blood culture-positive nosocomial sepsis, $15.7 \%$ (250 of 1588). The multivariate logistic regression analysis indicated that improved survival of EP infants was associated with discharged from specialist hospitals, hospitals located in high-level economic development region, increasing gestational age, increasing birth weight, antenatal steroids use and a history of premature rupture of membranes. However, twins or multiple births, Apgar $\leq 7$ at 5 min, cervical incompetence, and decision to withdraw care were associated with decreased survival.

Conclusions: Our study revealed the short-term outcomes of EP infants at discharge in China. The overall survival rate was lower than the developed countries, and medical care withdrawal was a serious problem. Nonetheless, improvements in care and outcomes have been made annually.

Keywords: Extremely preterm infant, Low birth weight, Survival rate, Complication, Outcome, China

\section{Background}

Extremely preterm (EP) infants with gestational age (GA) $<28$ weeks are at high risk of morbidity and mortality. Due to prematurity, they suffer higher incidence rate in respiratory distress syndrome (RDS), necrotizing enterocolitis (NEC), intraventricular hemorrhage (IVH), bronchopulmonary dysplasia (BPD), retinopathy of prematurity (ROP), infection and so on. Most of them need to receive advanced life support during the first several days. Consequently, the long-term survival rates were usually below 10\% before 1970 [1]. And, approximately one-quarter of EP infants born in the 1990s had a major disability at preschool age, such as impaired mental development, cerebral palsy, blindness, or deafness [2]. In recent decades, the outcomes of EP infants have been greatly improved due to tremendous advancement in perinatal care including antenatal steroids, surfactant replacement therapy, mechanical ventilation, nutrition therapy and increase of active treatment. From 2003 to 2007 , the median survival rates for infants born at 2227 weeks of gestation were $6,26,55,72,84$ and $88 \%$, respectively,in the United States [3]. Other studies from Netherlands, England, Canada, Switzerland and Australia demonstrated similar improvements as GA increased [4-8]. Obviously, these studies were completed in developed countries. However, outcomes of EP infants in China, a developing country with the greatest population, still remain unclear.

The available epidemiological data including shortterm and long-term outcomes of EP infants are very important to family counseling, clinical practice and social policies. Nevertheless, a population-based survey of EP infants in China is quite difficult to conduct because the exact number of EP infants is hard to evaluate. An infant born below 28 weeks of gestation is defined as an abortus, not as an infant, according to the current definition of preterm birth in China $[9,10]$. The number of EP infants died immediately in the delivery room due to lack of active and effective resuscitation, cannot be counted. Only those transferred to neonatal intensive care units (NICUs) after resuscitation can be known exactly. Thus, in this study, we focused solely on the short-term outcomes at discharge of EP infants from 26 NICUs in Guangdong province.

\section{Methods \\ Participating centers}

The collaborative study group composed of 26 NICUs was established before data collection. These NICUs were located in four regions of Guangdong province which were representative of medical units offering neonatal intensive care in the irrespective areas. The Third Affiliated Hospital of Guangzhou Medical University was responsible for coordinating this survey, where all the data were aggregated, stored and analyzed. This study was approved by the Ethics Committees of the Third Affiliated Hospital of Guangzhou Medical University. The same diagnostic criteria were applied to all NICUs.

\section{Subjects and data collection}

All EP infants discharged from our collaborative NICUs were studied, whereas live-born infants not admitted to NICUs and still births in the delivery room were not enrolled. The study protocol was fully discussed by all 
members, and a standardized questionnaire for data collection including maternal and neonatal demographics, major complications, treatments and outcomes was designed. In fact, this study was initiated at the end of 2012 and is still ongoing at present. Therefore, the data from January 1, 2008 to December 31, 2012 were collected retrospectively, and data from January 1, 2013 to December 31, 2017 were collected prospectively. The relevant records of all enrolled infants and their mothers were reviewed and filled in the questionnaire. All sheets were sent to the Third Affiliated Hospital of Guangzhou Medical University and the data from each questionnaire were input into the database. In order to minimize bias among centers and investigators, comprehensive and systematic training was provided to the staffs involved in the survey. Data collected by the researcher at each collaborative NICU was supervised and checked by the NICU director, who was responsible for the quality assurance. Meanwhile, these records were also checked for accuracy and completeness by collaborative centers.

\section{Definitions and classifications}

In this study, survivors were defined as neonates who survived to the time of discharge. GA was calculated from the date of the last menstrual period or was determined by fetal ultrasound assessment. RDS was diagnosed in preterm infants with the onset of respiratory distress shortly after birth and a compatible chest radiograph appearance [11]. BPD was defined as oxygen dependency at 36 weeks of corrected age or at discharge [12]. The criteria utilized in our survey for the diagnosis of NEC and for grading the severity of disease were based on Bell's stage [13]. ROP and the graded standard were defined by the international classification of ROP [14]. IVH and periventricular leukomalacia (PVL) were diagnosed by cranial ultrasonography or magnetic resonance imaging (MRI). The Papile grading system was used to grade IVH [15], and PVL was defined as degeneration of white matter adjacent to the cerebral ventricles following cerebral hypoxia or brain ischemia [16]. Nosocomial sepsis was defined as blood culture-positive sepsis occurring beyond $48 \mathrm{~h}$ of life [17].

\section{Statistical analysis}

All statistical analyses were performed using SPSS 18.0 for Windows (IBM, Armonk, NY, USA). Continuous variables were shown as means \pm standard deviation (SD) or as medians (P25, P75) when their distributions were highly skewed, which were analyzed using t-tests or MannWhitney tests. Categorical variables arepresented as rates and odds ratio with $95 \%$ confidence intervals (CI), which were analyzed using Chi-square tests. Multivariate analyses were performed by using logistic regression to analyze the risk factors of survival in preterm infants. $P<$ 0.05 was considered statistically significant.

\section{Results \\ Demographics of EP infants and mothers}

During the 10-year survey, in total, 2051 EP infants were enrolled. The overall survival rate at discharge was $52.5 \%$ (1076 of 2051). The smallest GA and lowest BW in survivors were 22 weeks and $480 \mathrm{~g}$ respectively. Totally, the mean GA was $26.68 \pm 1.00$ weeks, and the distribution ranged from $26(1.3 \%)$ for less than 24 weeks, 105 (5.1\%) for 24 weeks, 263 (12.8\%) for 25 weeks, 548 (26.7\%) for 26 weeks to 1109 (54.1\%) for 27 weeks. The mean BW was $935 \pm 179 \mathrm{~g}$, and the distribution ranged from $16(0.8 \%)$ for less than $500 \mathrm{~g}, 36(1.8 \%)$ for 500 $599 \mathrm{~g}, 106$ (5.2\%) for 600 - $699 \mathrm{~g}, 255$ (12.4\%) for 700 $799 \mathrm{~g}, 398(19.4 \%)$ for 800 - $899 \mathrm{~g}, 495$ (24.1\%) for 900 - $999 \mathrm{~g}$ to $745(36.3 \%)$ for equal or more than $1000 \mathrm{~g}$. In order to clarify the current treatment and outcome of EP infants, we specifically grouped the EP infants based on survival or not, as it was presented in Table 1. The overall mean GA or BW in the survivor group was greater than in the non-survivor group $(p<0.001)$. There was no significant difference in the sex ratio between the survivor and non-survivor group. There were fewer infants with Apgar scores $\leq 3$ at $1 \mathrm{~min}$ and $\leq 7$ at $5 \mathrm{~min}$ in the survivor group (both $p<0.001$ ). Comparing with the nonsurvivor group, the survivor group had higher rates of receiving surfactant therapy $(p<0.001)$ and non-invasive ventilation $(p<0.001)$, but the rate of requiring mechanical ventilation was lower $(p<0.01)$. Even more, there were significant differences in the days of mechanical ventilation, non-invasive ventilation, oxygen therapy and hospital stay between the two groups $(p<0.001)$.

For the mothers, there was a similar incidence in the history of pregnancy problems between the survivor and non-survivor groups. Comparing with the mothers in the non-survivor group, the mothers in the survivor group had a higher proportion of age $\geq 35$ years $(p<0.05)$ and antenatal steroids therapy $(p<0.001)$, while a lower rate of twin/multiple pregnancies $(p<0.05)$. Interestingly, the mothers in the survivor group had a higher incidence of premature rupture of membranes $(p<0.001)$ and a lower incidence of cervical incompetence $(p<0.01)$. However, there was no significant difference in the rate of cesarean section, or in the incidences of infection in the middle trimester of pregnancy, gestational diabetes mellitus, pregnancy-induced hypertension syndrome, placental abruption/placenta previa or thyroid dysfunction.

\section{Increase in number and survival rates of EP infants from 2008 to 2017}

The number of EP infants discharged from NICUs increased sharply from 58 cases in 2008 to 408 cases in 
Table 1 Demographics of extremely preterm (EP) infants and the mothers in outcome categories

\begin{tabular}{|c|c|c|c|c|c|}
\hline Characteristics & Survivors $(N=1076)$ & Non-survivors $(N=975)$ & Total $(N=2051)$ & $p$-value & OR $(95 \% C l)$ \\
\hline \multicolumn{6}{|l|}{ Characteristics of infants } \\
\hline GA (weeks), mean \pm SD & $26.92 \pm 0.86$ & $26.41 \pm 1.07$ & $26.68 \pm 1.00$ & $<0.001$ & / \\
\hline BW (grams), mean \pm SD & $976 \pm 167$ & $889 \pm 180$ & $935 \pm 179$ & $<0.001$ & / \\
\hline Gender (male), n (\%) & $669(62.2)$ & $586(60.1)$ & $1255(61.2)$ & $>0.05$ & 1.091 (0.913-1.303) \\
\hline \multicolumn{6}{|l|}{ Apgar score, n (\%) } \\
\hline$\leq 3$ at $1 \mathrm{~min}$ & $95(8.8)$ & $168(17.2)$ & $263(12.8)$ & $<0.001$ & $0.465(0.356-0.608)$ \\
\hline $4 \sim 7$ at $1 \mathrm{~min}$ & $369(34.3)$ & $391(40.1)$ & $760(37.1)$ & $<0.01$ & $0.780(0.651-0.933)$ \\
\hline$\leq 3$ at $5 \mathrm{~min}$ & $9(0.8)$ & $43(4.4)$ & $52(2.5)$ & $<0.001$ & $0.183(0.089-0.377)$ \\
\hline $4 \sim 7$ at $5 \mathrm{~min}$ & $130(12.1)$ & $214(21.9)$ & $344(16.8)$ & $<0.001$ & $0.489(0.385-0.620)$ \\
\hline Surfactant therapy, n (\%) & 900 (83.6) & 718 (73.6) & $1618(78.9)$ & $<0.001$ & $1.830(1.476-2.270)$ \\
\hline Surfactant therapy twice or more, $\mathrm{n}(\%)$ & $155(14.4)$ & $155(15.9)$ & $310(15.1)$ & $>0.05$ & $0.890(0.699-1.134)$ \\
\hline Mechanical ventilation, n (\%) & $816(75.8)$ & $787(80.7)$ & $1603(78.2)$ & $<0.01$ & $0.750(0.607-0.926)$ \\
\hline $\begin{array}{l}\text { Days of mechanical ventilation (days), } \\
\text { median (P25, P75) }\end{array}$ & $8.0(2.0,22.0)$ & $2.3(1.0,7.3)$ & $4.2(1.0,15.0)$ & $<0.001$ & / \\
\hline Non-invasive ventilation, $\mathrm{n}(\%)$ & $880(81.8)$ & $240(24.6)$ & $1120(54.6)$ & $<0.001$ & $13.750(11.117-17.007)$ \\
\hline $\begin{array}{l}\text { Days of non-invasive ventilation (days), } \\
\text { median (P25, P75) }\end{array}$ & $15.4(6.0,26.0)$ & $3.1(1.0,8.0)$ & $12.0(4.0,23.0)$ & $<0.001$ & / \\
\hline $\begin{array}{l}\text { Total days of oxygen therapy (days), } \\
\text { median (P25, P75) }\end{array}$ & $42.7(25.0,60.6)$ & $3.0(1.0,9.8)$ & $19.5(2.8,47.0)$ & $<0.001$ & / \\
\hline $\begin{array}{l}\text { Length of hospital stay (days), } \\
\text { median (P25, P75) }\end{array}$ & $71.0(55.5,87.0)$ & $3.0(1.0,10.0)$ & $35.0(3.0,73.0)$ & $<0.001$ & / \\
\hline \multicolumn{6}{|l|}{ Characteristics of mothers } \\
\hline History of pregnancy problems ${ }^{a}, \mathrm{n}(\%)$ & $412(38.3)$ & $349(35.8)$ & $761(37.1)$ & $>0.05$ & $1.113(0.930-1.332)$ \\
\hline Age $\geq 35$ years, $n(\%)$ & $238(22.1)$ & $177(18.2)$ & $415(20.2)$ & $<0.05$ & $1.280(1.030-1.591)$ \\
\hline Cesarean section, n (\%) & $221(20.5)$ & $172(17.6)$ & $393(19.2)$ & $>0.05$ & $1.207(0.967-1.506)$ \\
\hline Twin/multiple pregnancy, n (\%) & $392(36.4)$ & $404(41.4)$ & $796(38.8)$ & $<0.05$ & $0.810(0.678-0.968)$ \\
\hline Antenatal steroids, n (\%) & $609(56.6)$ & 367 (37.6) & $976(47.6)$ & $<0.001$ & $2.160(1.810-2.579)$ \\
\hline Dexamethasone $\geq 4$ doses & $390(36.2)$ & $204(20.9)$ & $594(29.0)$ & $<0.001$ & $2.149(1.762-2.620)$ \\
\hline Dexamethasone 1 3 dose(s) & $219(20.4)$ & $163(16.7)$ & $382(18.6)$ & $<0.05$ & $1.273(1.017-1.593)$ \\
\hline Premature rupture of membranes, $n(\%)$ & $305(28.3)$ & $194(19.9)$ & $499(24.3)$ & $<0.001$ & $1.593(1.297-1.956)$ \\
\hline $\begin{array}{l}\text { Infection in the middle trimester of } \\
\text { pregnancy, } \mathrm{n}(\%)\end{array}$ & $82(7.6)$ & $66(6.8)$ & $148(7.2)$ & $>0.05$ & $1.136(0.812-1.590)$ \\
\hline Gestational diabetes mellitus, n (\%) & $99(9.2)$ & $88(9.0)$ & $187(9.1)$ & $>0.05$ & $1.021(0.756-1.380)$ \\
\hline Pregnancy induced hypertension syndrome, n (\%) & $71(6.6)$ & $80(8.2)$ & $151(7.4)$ & $>0.05$ & $0.790(0.567-1.102)$ \\
\hline Placental abruption/Placenta previa, n (\%) & $72(6.7)$ & $81(8.3)$ & $153(7.5)$ & $>0.05$ & $0.792(0.569-1.101)$ \\
\hline Thyroid dysfunction, n (\%) & $31(2.9)$ & $22(2.3)$ & $53(2.6)$ & $>0.05$ & $1.285(0.739-2.235)$ \\
\hline Cervical incompetence, n (\%) & $28(2.6)$ & $48(4.9)$ & $76(3.7)$ & $<0.01$ & $0.516(0.321-0.829)$ \\
\hline
\end{tabular}

$P$-value means the contrast between survivors and non-survivors

GA gestational age, $B W$ birth weight, SD standard deviation, $P 25$ the 25 th percentile, $P 75$ the 75 th percentile, $O R$ odds ratio, $C l$ confidence interval

${ }^{a}$ History of pregnancy problems refer to that the mother had at least one of the histories as follow: spontaneous abortion, induced abortion, stillbirth, preterm

birth, ectopic pregnancy, or baby died during the neonatal period

2017, as it was shown in Table 2. The survival rate improved annually from $36.2 \%$ in 2008 to $59.3 \%$ in 2017 $(p<0.001)$. The ratio of EP infants to all preterm infants rose annually from $0.70 \%$ in 2008 to $2.29 \%$ in 2017 ( $p<$ 0.001). Similarly, the ratio of EP infants to all discharged infants increased annually from $0.17 \%$ in 2008 to $0.67 \%$ in $2017(p<0.001)$.
Improved survival rates of EP infants with increasing GA and BW from 2008 to 2017

For the entire cohort, with the increase in GA or BW, the number of EP infants increased from $26(\mathrm{GA}<24$ weeks) or $52(\mathrm{BW}<600 \mathrm{~g})$ to $1109(\mathrm{GA}=27$ weeks) or 745 (BW $\geq 1000 \mathrm{~g}$ ), as it was displayed in Table 3. As GA increased from less than 24 weeks to 27 weeks, the 
Table 2 The survival rate of extremely preterm (EP) infants at discharge from 2008 to 2017

\begin{tabular}{|c|c|c|c|c|c|c|c|c|c|c|c|}
\hline & 2008 & 2009 & 2010 & 2011 & 2012 & 2013 & 2014 & 2015 & 2016 & 2017 & Total \\
\hline All EP infants, $n$ & 58 & 75 & 78 & 158 & 163 & 186 & 257 & 299 & 369 & 408 & 2051 \\
\hline Survived, n (\%) & $21(36.2)$ & 25 (33.3) & $28(35.9)$ & $66(41.8)$ & 79 (48.5) & $95(51.1)$ & $\begin{array}{l}130 \\
(50.6)\end{array}$ & $\begin{array}{l}170 \\
(56.9)\end{array}$ & $\begin{array}{l}220 \\
(59.6)\end{array}$ & $\begin{array}{l}242 \\
(59.3)\end{array}$ & $\begin{array}{l}1076 \\
(52.5)\end{array}$ \\
\hline $\begin{array}{l}\text { Died despite active } \\
\text { treatment, } \mathrm{n}(\%)\end{array}$ & $14(24.1)$ & $16(21.3)$ & $12(15.4)$ & $33(20.9)$ & $16(9.8)$ & $22(11.8)$ & $54(21.0)$ & $58(19.4)$ & $52(14.1)$ & $44(10.8)$ & $321(15.7)$ \\
\hline $\begin{array}{l}\text { Died after medical } \\
\text { care withdrawal, n (\%) }\end{array}$ & $23(39.7)$ & $34(45.3)$ & $38(48.7)$ & $59(37.3)$ & $68(41.7)$ & 69 (37.1) & $73(28.4)$ & $71(23.7)$ & 97 (26.3) & $\begin{array}{l}122 \\
(29.9)\end{array}$ & 654 (31.9) \\
\hline $\begin{array}{l}\text { All preterm infants, } \\
\mathrm{n}(\%)^{*}\end{array}$ & $\begin{array}{l}8335 \\
(0.70)\end{array}$ & $\begin{array}{l}8499 \\
(0.88)\end{array}$ & $\begin{array}{l}10,795 \\
(0.72)\end{array}$ & $\begin{array}{l}14,210 \\
(1.11)\end{array}$ & $\begin{array}{l}15,611 \\
(1.04)\end{array}$ & $\begin{array}{l}16,574 \\
(1.12)\end{array}$ & $\begin{array}{l}16,266 \\
(1.58)\end{array}$ & $\begin{array}{l}15,967 \\
(1.87)\end{array}$ & $\begin{array}{l}18,417 \\
(2.00)\end{array}$ & $\begin{array}{l}17,780 \\
(2.29)\end{array}$ & $\begin{array}{l}142,454 \\
(1.44)\end{array}$ \\
\hline All infants, $\mathrm{n}(\%)^{\#}$ & $\begin{array}{l}33,299 \\
(0.17)\end{array}$ & $\begin{array}{l}33,328 \\
(0.22)\end{array}$ & $\begin{array}{l}43,305 \\
(0.18)\end{array}$ & $\begin{array}{l}53,066 \\
(0.29)\end{array}$ & $\begin{array}{l}58,718 \\
(0.28)\end{array}$ & $\begin{array}{l}55,603 \\
(0.33)\end{array}$ & $\begin{array}{l}53,951 \\
(0.48)\end{array}$ & $\begin{array}{l}53,937 \\
(0.55)\end{array}$ & $\begin{array}{l}60,075 \\
(0.61)\end{array}$ & $\begin{array}{l}60,886 \\
(0.67)\end{array}$ & $\begin{array}{l}506,168 \\
(0.41)\end{array}$ \\
\hline
\end{tabular}

$\mathrm{R} \times \mathrm{C}$ Chi-square test (linear-by-linear association) showed that the ratios of EP infants in all preterm infants and in all infants discharged rose up annually (both $P<$ 0.001). And the annual survival rate of EP infants improved from 2008 to $2017(P<0.001)$. EP: Extremely preterm; ${ }^{*}$ Percentile of EP infants discharged in all preterm infants discharged; "Percentile of EP infants discharged in all infants discharged

survival rate improved from 11.5 to $62.4 \%(p<0.001)$. As $\mathrm{BW}$ increased from less than $600 \mathrm{~g}$ to above $1000 \mathrm{~g}$, the survival rate rose dramatically from 23.1 to $64.2 \%(p<$ 0.001). There was only one infant who survived with BW less than 500 g.However, there were no significant differences in GA or BW between 2008-2012 and 2013$2017(p=0.073$ or $p=0.500$ ). The mean GA or BW for EP infants during 2008-2012 were $26.73 \pm 0.93$ weeks or $935 \pm 172$ g respectively, while during 2013-2017 were $26.66 \pm 1.02$ weeks or $935 \pm 181$ g respectively.

\section{Variations in survival rates among different regions and hospitals}

According to the level of economic development where the hospitals were located, our collaborative NICUs could be divided into three categories, specifically involving Guangzhou and Shenzhen (including 11 hospitals) with high level, the cities in the Pearl Delta (including 10 hospitals) with medium level and the cities outside the Pearl Delta (including 5 hospitals) with low level. As it was shown in Table 4, from 2008 to 2017, the overall survival rates were positively correlated with the level of regional economic development, and there were significant differences among these regions $(p<$ 0.001). From the low to high level, the mean GA of EP infants was $26.74 \pm 0.90$ weeks, $26.79 \pm 0.92$ weeks and
$26.62 \pm 1.04$ weeks, which showed a significant difference $(p=0.002)$; while the mean BW was $920 \pm 174 \mathrm{~g}, 947 \pm$ $170 \mathrm{~g}$ and $932 \pm 183 \mathrm{~g}$, which showed no significant difference $(p=0.104)$.

Among the 26 hospitals, 19 were general hospitals and the others were specialist hospitals (maternal and children's hospitals), and the overall survival rate of EP infants discharged from general hospitals was lower than specialist hospitals (OR $=0.530,95 \%$ CI: $0.445-0.632$, $p<0.001)$. Nevertheless, the mean GA and BW of EP infants from general hospitals $(26.75 \pm 0.94$ weeks and $944 \pm 177$ g respectively) were both greater than those from specialist hospitals $(26.60 \pm 1.05$ weeks and $924 \pm$ $180 \mathrm{~g}$ respectively), and there were significant differences $(p=0.001$ and $p=0.013)$.

\section{Complications of EP infants}

RDS was the most common complication in EP infants, who were diagnosed with RDS accounted for $88.0 \%$ (1804 of 2051). Therefore, the proportion of EP infants receiving surfactant therapy and mechanical ventilation was $78.9 \%$ (1618 of 2051) and 78.2\% (1603 of 2051) respectively, as shown in Table 1.There was no significant difference in RDS incidence among GA groups as shown in Fig. 1. BPD was another serious complication in EP infants. In this survey, $68.0 \%$ (788 of 1158) of EP infants

Table 3 The survival rate of extremely preterm (EP) infants in relation to gestational age and birth weight

\begin{tabular}{|c|c|c|c|c|c|c|c|c|c|c|c|}
\hline & \multicolumn{5}{|c|}{ GA (weeks) } & \multicolumn{6}{|c|}{ BW (grams) } \\
\hline & $<24$ & $24 \sim$ & $25 \sim$ & $26 \sim$ & $27 \sim$ & $<600$ & $600 \sim$ & $700 \sim$ & $800 \sim$ & $900 \sim$ & $\geq 1000$ \\
\hline All EP infants, $n$ & 26 & 105 & 263 & 548 & 1109 & 52 & 106 & 255 & 398 & 495 & 745 \\
\hline Survived, n (\%) & $3(11.5)$ & $36(34.3)$ & 89 (33.8) & $256(46.7)$ & $692(62.4)$ & $12(23.1)$ & $32(30.2)$ & 86 (33.7) & $186(46.7)$ & $282(57.0)$ & $478(64.2)$ \\
\hline $\begin{array}{l}\text { Died despite active } \\
\text { treatment, } \mathrm{n}(\%)\end{array}$ & $8(30.8)$ & $23(21.9)$ & $56(21.3)$ & $90(16.4)$ & $144(13.0)$ & $11(21.2)$ & $33(31.1)$ & $41(16.1)$ & $66(16.6)$ & $74(14.9)$ & $96(12.9)$ \\
\hline $\begin{array}{l}\text { Died after medical care } \\
\text { withdrawal, n (\%) }\end{array}$ & $15(57.7)$ & $46(43.8)$ & $118(44.9)$ & $202(36.9)$ & $273(24.6)$ & $29(55.8)$ & 41 (38.7) & $128(50.2)$ & $146(36.7)$ & $139(28.1)$ & $171(23.0)$ \\
\hline
\end{tabular}

$\mathrm{R} \times 2$ Chi-square test (linear-by-linear association) showed that the survival rate of EP infants improved with increasing GA and BW (both $P<0.001$ ). EP extremely preterm, GA gestational age, $B W$ birth weight 
Table 4 Differences of survival rate among regions and between hospitals

\begin{tabular}{|c|c|c|c|c|c|}
\hline & \multicolumn{3}{|c|}{ Economic development levels of the region } & \multicolumn{2}{|l|}{ Types of hospital } \\
\hline & High-level & Middle-level & Low-level & General hospitals & Maternal and children's hospitals \\
\hline All EP infants, $n$ & 1274 & 559 & 218 & 1083 & 968 \\
\hline Survived, n (\%) & $708(55.6)$ & $281(50.3)$ & $87(39.9)$ & $488(45.1)$ & $588(60.7)$ \\
\hline Died despite active treatment, $\mathrm{n}(\%)$ & $182(14.3)$ & $81(14.5)$ & $58(26.6)$ & $213(19.7)$ & $108(11.2)$ \\
\hline Died after medical care withdrawal, n (\%) & $384(30.1)$ & $197(35.2)$ & $73(33.5)$ & $382(35.3)$ & $272(28.1)$ \\
\hline
\end{tabular}

$\mathrm{R} \times 2$ Chi-square test (linear-by-linear association) showed that the overall survival rate of EP infants discharged from different regions improved with the ascending level of economic development $(P<0.001)$. And $2 \times 2$ Chi-square test showed that the survival rate of EP infants discharged from general hospitals was lower than those from maternal and children's hospitals $(P<0.001)$. EP: Extremely preterm

were diagnosed with oxygen dependency on postnatal day 28 , but only $32.3 \%$ (374 of 1158 ) still required oxygen therapy at 36 weeks of corrected age or at discharge. The incidence of BPD decreased significantly with the increase of GA $(p<0.001) .1117$ EP infants underwent eye examination, $504(45.1 \%)$ of whom were diagnosed with ROP at different stages, and the proportion of stage $\geq 3$ was $14.6 \%$ (163 of 1117). $88 \mathrm{EP}$ infants received further treatments, 40 of whom underwent intravitreal injection with anti-VEGF. The incidence of ROP declined with the increase of GA $(p<0.001)$. NEC was evaluated in EP infants who survived for more than $48 \mathrm{~h} .10 .1 \%$ (160 of 1588) of EP infants met the diagnostic criteria at any stage of NEC, 27 of whom required surgery. The incidence of NEC appeared to be positive with GA $(p<$ 0.05). $1431 \mathrm{EP}$ infants were examined by head ultrasound or MRI, of whom 37.4\% (535 of 1431) were diagnosed with IVH, 13.3\% (190 of 1431) of EP infants were diagnosed with severe IVH at grade $\geq 3$. The incidence of IVH was inversely related to gestational age $(p<$
0.001). The incidence of PVL was $6.2 \%$ ( 89 of 1431), and there was no significant difference among $\mathrm{GA}<25$ weeks, $\mathrm{GA}=25$ weeks, $\mathrm{GA}=26$ weeks and $\mathrm{GA}=27$ weeks. Among the 1588 infants survived more than $48 \mathrm{~h}$, 250 infants $(15.7 \%)$ were diagnosed with blood culturepositive nosocomial sepsis. The complications for each subgroup of GA are shown in Fig. 1. Additionally, comparing with the period of 2008-2012, the incidence of RDS and BPD increased sharply during the period of 2013-2017(OR $=2.076,95 \%$ CI: $1.574-2.738, p<0.001$; $\mathrm{OR}=1.645$, 95\% CI: $1.152-2.351, p<0.01)$. On the contrary, the incidence of PVL decreased dramatically (OR = 0.252 , 95\% CI: $0.163-0.392, p<0.001)$. Moreover, the incidence of ROP, NEC and IVH did not change significantly. The results were shown in Fig. 2.

\section{Survival days of the non-survivors}

Among the 975 non-survivors, 893 (91.6\%) of EP infants died during the neonatal period ( $\leq 28$ days), the other 82 (8.4\%) died after the neonatal period ( $>28$ days) as it was

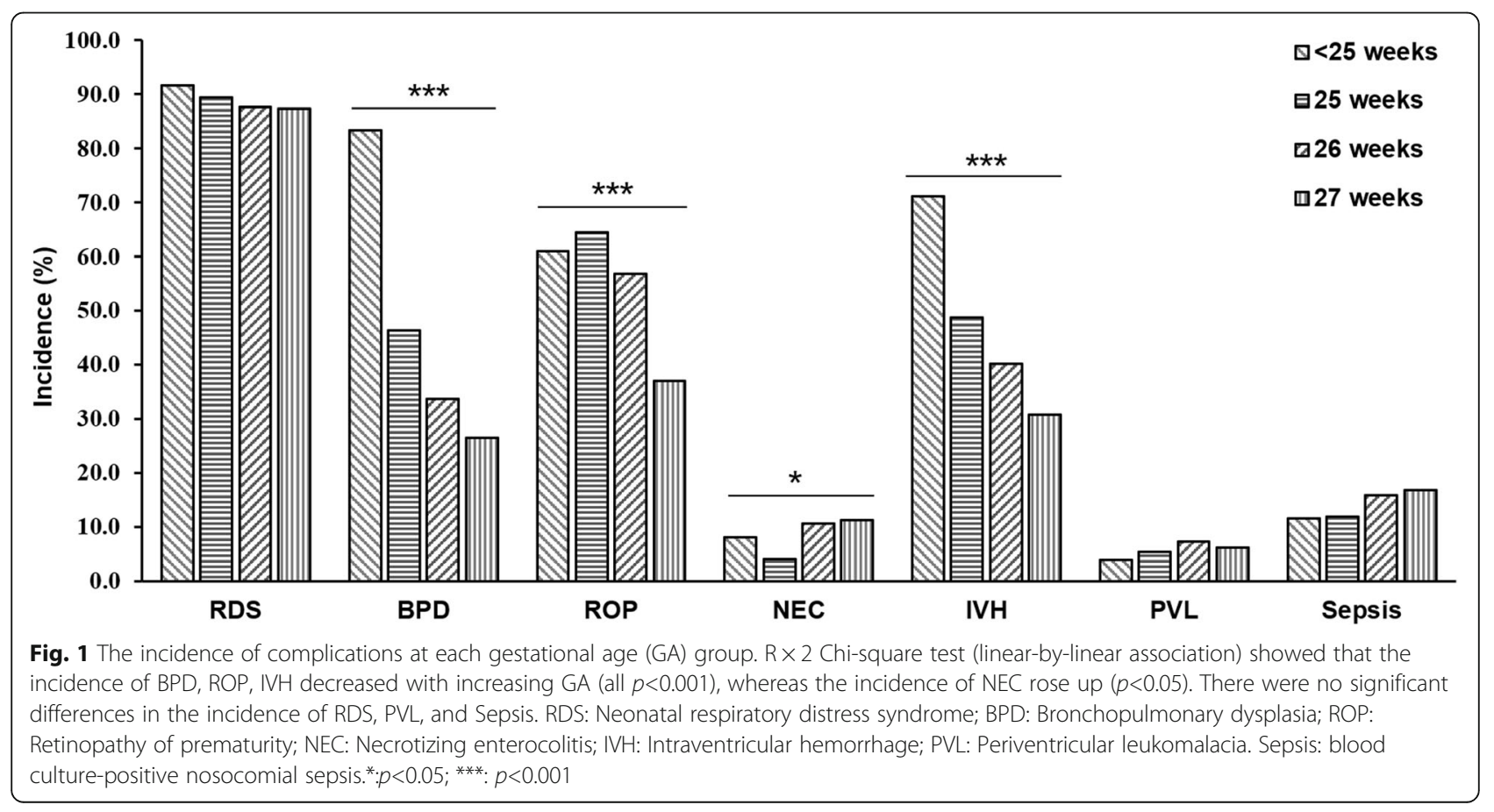




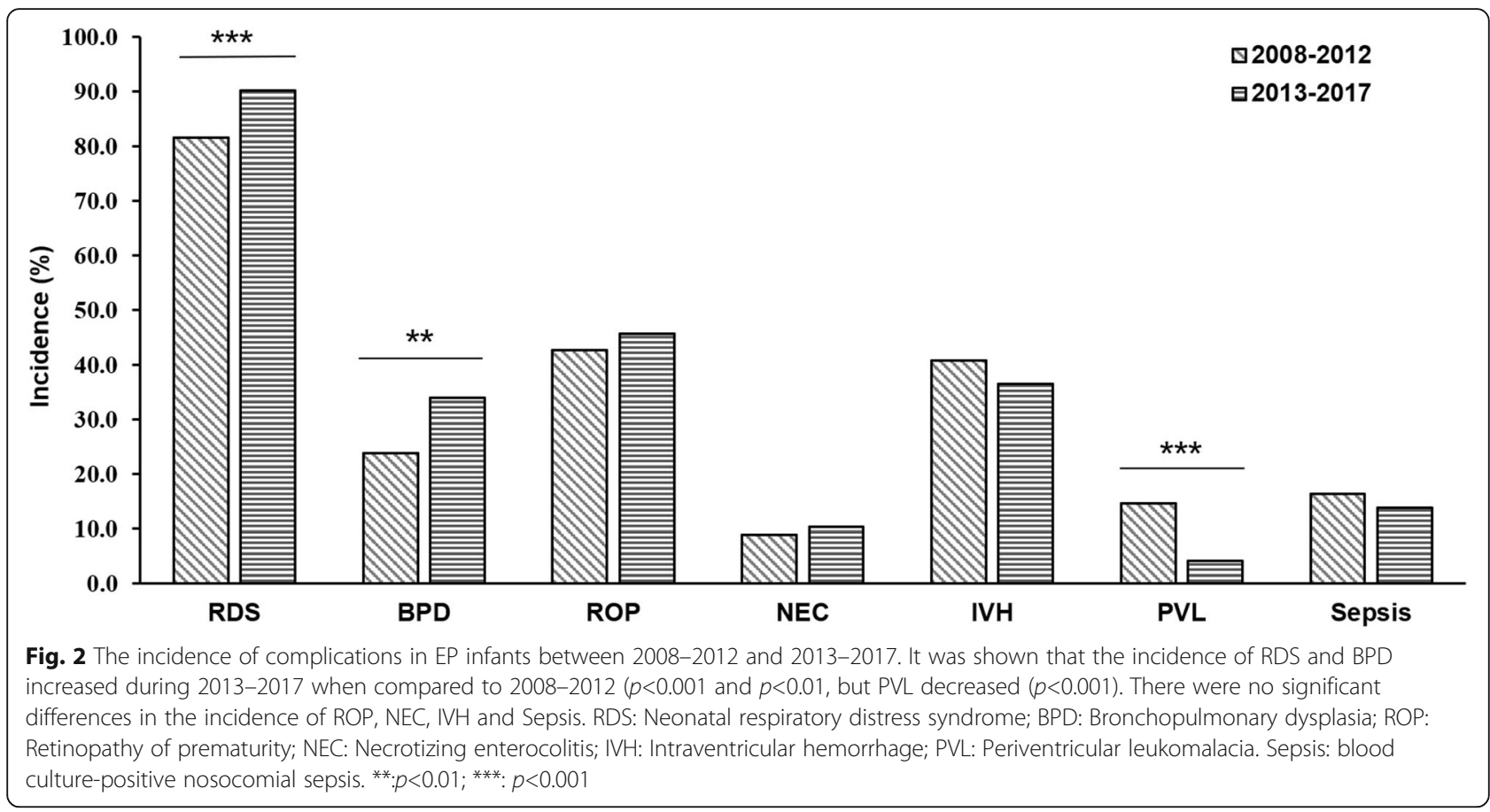

shown in Fig. 3. Specifically, 324 (33.2\%) of EP infants died on the first day, $139(14.3 \%)$ on the second day, 88 (9.0\%) on the third day, $129(13.2 \%)$ during the fourth to seven day, 97 (9.9\%) in the second week, $71(7.3 \%)$ in the third week and 45 (4.6\%) in the fourth week. The survival days of non-survivors under active treatment or medical care withdrawal were shown in Fig. 3. The chisquare test showed that there was a significant difference in the distribution of survival days between the two groups $(p<0.001)$. No significant difference in the distribution of survival days of non-survivors was found among the different GA (GA $<25$ weeks, GA $=25$ weeks, $\mathrm{GA}=26$ weeks or $\mathrm{GA}=27$ weeks) by chi-square test.

In our survey, 654 EP infants died mainly as a result of medical care withdrawal. The main reasons for the decision to withdraw care were summarized, as they were shown in Fig. 4. The most important reason was the economic burden and fearing of poor or uncertain outcomes, followed by only fearing of poor or uncertain outcomes, only concerning about economic burden, unknown reason and other factors, which was accounted for $34.4,33.0,19.0,10.7$ and $2.7 \%$ respectively.

\section{Multivariate logistic regression analysis of risk factors for survival}

As it was shown in Table 5, the multivariate logistic regression analysis indicated that improved survival of EP infants was associated with discharged from specialist hospital, hospitals located in high-level economic development region, increasing gestational age, increasing birth weight, antenatal steroids use and a history of premature rupture of membranes. On the contrary, twins or multiple births, Apgar $\leq 7$ at $5 \mathrm{~min}$, cervical incompetence and decision to withdraw care were associated with decreased survival.

\section{Discussion}

Our study revealed for the first time the short-term outcomes of EP infants in China over 10 years, which provided useful data to gain insight into the current status of preterm infants. A report from WHO [18] declared that preterm birth had become a growing global health issue that the number of preterm infants born worldwide was nearly 15 million annually and continued to grow, and the number of premature babies from China ranked in the second. Despite the encouraging progress, more than a million of preterm infants died mainly because of severe complications associated with premature birth. Prematurity has become the leading cause of death in the first month of life [19]. The Canada Neonatal Network $(\mathrm{CNN})$ data demonstrated substantially decrease in the mortality rate of GA < 29 weeks from $17.2 \%$ (19961997) to $14.7 \%$ (2006-2007) [6]. Isayama $\mathrm{T}$, et al. had compared Neonatal Research Network of Japan (NRNJ) data with CNN data during 2006-2008, the mortality rate at GA $<25$ weeks, $26-27$ weeks, $28-29$ weeks and $30-32$ weeks were $27.1 \%$ vs $52.3 \%, 9.6 \%$ vs $17.9 \%, 4.1 \%$ vs $7.3 \%$ and $1.4 \%$ vs $1.7 \%$ [20]. EP infants with a GA less than 28 weeks are at higher risk of morbidity and mortality. In our survey, the overall mortality of EP infants at discharge was $47.5 \%$ during 2008-2017. Specifically, the mortality rate at $\mathrm{GA}<24$ weeks, 24 weeks, 25 weeks, 


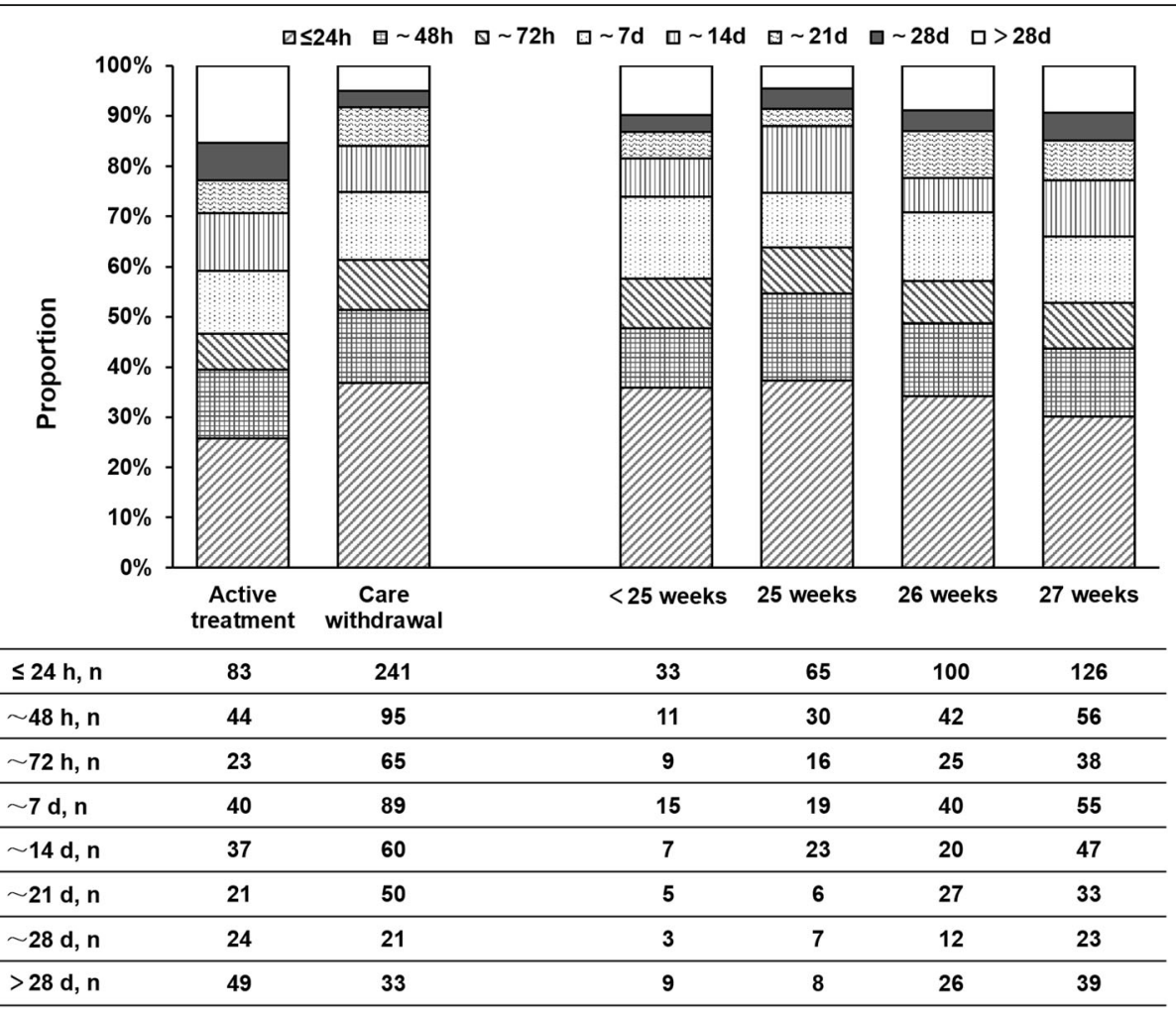

Fig. 3 The survival days (hours) of non-survival EP infants. The left part of the chart showed the duration of survival in the non-survival EP infants under active treatment or care withdrawal. The right part of the chart showed the duration of survival in the non-survival EP infants at different GA

26 weeks, and 27 weeks were $88.5,65.7,66.2,53.3$, and $37.6 \%$ respectively. Although the mortality declined with GA increase, it was obviously higher than in developed countries. It seems that these results may be frustrating, but we were encouraged by the improvement in annual survival rate from $36.2 \%(21 / 58)$ in 2008 to $59.3 \%$ (242/ 408) in 2017.

Due to the definition of preterm birth in China as mentioned above, parents currently can decide whether babies receive treatments or not. Many EP infants died as a result of medical care withdrawal, which accounted for $67.1 \%$ in non-survivors of EP infants and $31.9 \%$ in total EP infants. What were the factors that influenced the parental decision? Just like in many developing countries, the most important factor may be an economic burden. It was reported recently that cost was indeed an important factor influencing active management of EP infants [21]. As it was indicated in our survey, EP infants in the cities with high level of economic development had low mortality due to medical care withdrawal, such as Guangzhou or Shenzhen. With reference to the annual per capita net income, medical costs for EP infants were a huge burden for families especially in regions of low-level economic development such as cities outside the Pearl Delta. Optimistically, in recent years with an increase in family income and improvement of social security system the survival rate of premature infants has greatly improved.

Moreover, fear of poor or uncertain outcomes in EP infant was another important factor affecting the parental decision. Owing to extreme prematurity, EP infants have a higher incidence of complications, and maybe develop serious consequences in the future [22]. Numerous studies have suggested that major neonatal morbidities are associated with a higher incidence of adverse neurodevelopmental outcomes [23-25]. Even worse, due to lack of large samples of epidemiological data for these infants, clinicians or parents were often in the dilemma to make timely and accurate decisions. When expectations were not met or a foreseeable adverse event emerged, parents' confidence may be shaken and thus the original active treatment options may be changed. In addition, under the influence of outmoded conventional ideas and the only-child policy (the most important part of Family Planning Policy in China), many Chinese families had a preference for male babies, which led to care withdrawal for female infants. In our study, the proportion of male babies was greater than females $(61.2 \% \mathrm{vs}$ $38.9 \%)$, but no significant difference was found between the survivor and non-survivor groups. 


\section{口Economic burden \\ $\square$ Fearing of poor or uncertain outcomes \\ Economic burden and fearing of poor or uncertain outcomes ๑Other factors \\ 口Unknow}

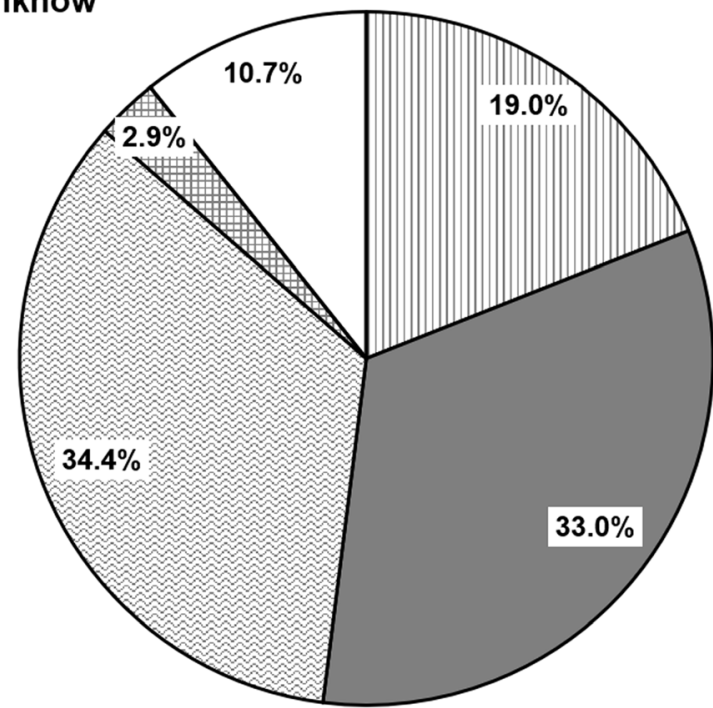

Fig. 4 The reasons for medical care withdrawal. Economic burden and fearing of poor or uncertain outcomes, only fearing of poor or uncertain outcomes, only concerning about economic burden, unknow and other factors were the main reasons leading to medical care withdrawal, which was accounted for $34.4,33.0,19.0,10.7$ and $2.9 \%$ respectively

Extremely preterm birth survivors exhibited significant morbidity. Many studies showed the major neonatal morbidities are predictive of long-term disorders such as motor impairment, cognitive disorders, behavior problems, poor general health, hearing loss, and visual problem. Our study found that the major complications during hospitalization were RDS (88.0\%), BPD (32.3\%), ROP (45.1\%), NEC (10.1\%), IVH (37.4\%), PVL (6.2\%) and blood culture-positive nosocomial sepsis (15.7\%). In fact, the incidence of these complications may be much higher than we imagined. There were a considerable number of infants died on account of medical care withdraw whose complications did not develop in our survey. Besides, the diagnosis of some complications may be missed due to lack of relevant equipment for examinations or follow-up data after discharge, especially in the region with low-level economic development. For example, the diagnosis of IVH and PVL might have been missed because they were not checked by head ultrasound or MRI in time. Thus, these results should be interpreted with caution when compared to other studies.

Multiple factors were associated with neonatal mortality and morbidity. A systematic review and meta-analysis

Table 5 Multivariate logistic regression analysis of risk factors for survival

\begin{tabular}{lllll}
\hline Influencing factors & $\beta$ value & Wald $x^{2}$ & $p$ & OR (95\% Cl) \\
\hline Specialist hospital & 0.820 & 35.771 & $<0.001$ & $2.270(1.735-2.969)$ \\
Economic development in high-level & 0.301 & 4.958 & 0.026 & $1.351(1.037-1.762)$ \\
Gestational age & 0.405 & 25.770 & $<0.001$ & $1.499(1.282-1.753)$ \\
Birth weight & 0.001 & 10.020 & 0.002 & $1.001(1.001-1.002)$ \\
Twins or multiple births & -0.316 & 5.288 & 0.021 & $0.729(0.557-0.954)$ \\
Apgar $\leq 7$ at 5 min & -0.714 & 19.088 & $<0.001$ & $0.490(0.356-0.675)$ \\
Antenatal steroids & 0.493 & 13.999 & $<0.001$ & $1.637(1.265-2.119)$ \\
Premature rupture of membranes & 0.367 & 5.424 & 0.020 & $1.444(1.060-1.967)$ \\
Cervical incompetence & -0.667 & 4.149 & 0.042 & $0.513(0.270-0.975)$ \\
Care withdrawal & -4.490 & 400.618 & $<0.001$ & $0.011(0.007-0.017)$ \\
\hline
\end{tabular}


showed that antenatal corticosteroid therapy could reduce perinatal death and the incidence of RDS, IVH and NEC in preterm infants [26]. In our study, the survivor group had a higher rate of antenatal corticosteroid therapy than the non-survivor group, but the overall rate of antenatal corticosteroid therapy was lower than other studies [27, 28]. Therefore, prenatal management should be strengthened in the future. In addition, it was indicated in our study that discharged from specialist hospital, discharged hospitals located in high-level economic development region, increasing gestational age, increasing birth weight, antenatal steroids use and a history of premature rupture of membranes were associated with improved survival of EP infants. Instead, twins or multiple births, Apgar $\leq 7$ at $5 \mathrm{~min}$, cervical incompetence and decision to care withdrawal were associated with decreased survival.

Although the total number of subjects in our study was limited, it still represents the short-term outcomes of EP infants in Guangdong province in China. This study was also the first multicenter survey on outcomes of EP infants in China. Therefore, our study may help to delineate the current survival rate of EP infants in China and thus serve as a benchmark for future investigations and studies. Of course, there were still some limitations to our study. First, our survey included only 26 large tertiary hospitals and was neither population- based nor a nationwide study. Second, it only focused on short-term outcomes at discharge and did not consider long term outcomes, especially in neural developmental disabilities.

With societal advancement, economic development and improvements in medicine, as well as adjustments in the Family Planning Policy, the preterm birth has now become an important issue in China. More and more extremely premature infants will be born and survive. To facilitate this, a clear and unified clinical classification and guideline for EP infants should be developed as soon as possible. As in some developed countries, the minimum GA for the definition of preterm birth should be 20-22 weeks. Newborns older than 23 weeks of gestation should routinely receive resuscitation, and even those under 21-22 weeks should be treated similarly if parents insist [29].

\section{Conclusions}

Our study revealed the short-term outcomes of EP infants at discharge among a large population in China for the first time. The survival rate of these infants was lower when compared to developed countries, and medical care withdrawal was a serious dilemma for these infants. Thus, it is crucial to carry on population- based and nationwide research on EP infants in order to develop new clinical practice guidelines for them.

\section{Abbreviations}

BPD: Bronchopulmonary dysplasia; BW: Birth weight; EP: Extremely preterm; GA: Gestational age; IVH: Intraventricular hemorrhage; MRI: Magnetic resonance imaging; NEC: Necrotizing enterocolitis; NICUs: Neonatal intensive care units; PVL: Periventricular leukomalacia; RDS: Respiratory distress syndrome; SD: Standard deviation

\section{Acknowledgments \\ The authors gratefully acknowledge all the institutes and investigators.}

\section{Authors' contributions}

FW, GSL and QLC conceptualized the study, analyzed the data, and wrote the manuscript. ZSF, CZY, YHD, XZY, LD, HXC, ZZ, WS, BYY, LYH, HPR, YY, FFW, SGD, QM, BW, YGH, WZL, YLC, ZFC, XYL2, NYL, SSH, ZHW, BB and YXY helped with the design, data collection and analysis of the study, and revised drafts of the manuscript. XHT, XTY, WYL, JM, BQW, CWL, XR, WMH, $J F L, H W H, W K Y, X J R, D L, X Y L 1, Y W, L Z, D A, J L C, W L, Y Q D, Y F H, Y F C, Y J, Y B$ and $\mathrm{GHL}$ helped collect data and review drafts of the manuscript. All authors have read and approved the final manuscript.

\section{Funding}

This work was supported by Guangzhou Science Technology and Innovation Grants (2014Y2-00053 to QLC), and Guangdong Science and Technology Department Grants (2017ZC0254 to FW). The funder had no role in study design, data collection and analysis, decision to prepare or publish the manuscript.

\section{Availability of data and materials}

The datasets used and/or analyzed during the current study are available from the corresponding author on reasonable request.

\section{Ethics approval and consent to participate}

Data collection was approved by the Institutional Review Board of the Third Affiliated Hospital of Guangzhou Medical University. Written consents of parents were obtained at the time of admission.

Consent for publication

Not applicable.

\section{Competing interests}

The authors declare that they have no competing interests.

\section{Author details}

${ }^{1}$ Department of Neonatology, the First Affiliated Hospital of Jinan University, Guangzhou 510630, Guangdong, China. ${ }^{2}$ Department of Pediatrics, the Third Affiliated Hospital of Guangzhou Medical University, Guangzhou 510150, Guangdong, China. ${ }^{3}$ Department of Neonatology, Shenzhen Maternal \& Child Healthcare Hospital, Affiliated Southern Medical University, Shenzhen 518028, Guangdong, China. ${ }^{4}$ Department of Neonatology, Foshan Maternal and Child's Hospital, Foshan 528000, Guangdong, China. ${ }^{5}$ Department of Neonatology, Women and Children Hospital of Guangdong Province, Guangzhou 510010, Guangdong, China. ${ }^{6}$ Department of Neonatology, Shenzhen People's Hospital, Shenzhen 518020, Guangdong, China.

${ }^{7}$ Department of Neonatology, Meizhou People's Hospital, Meizhou 514031, Guangdong, China. ${ }^{8}$ Department of Neonatology, Guangzhou Women and Children's Medical Center, Guangzhou 510120, Guangdong, China. ${ }^{9}$ Department of Neonatology, Nanfang Hospital of Southern Medical University, Guangzhou 510515, Guangdong, China. ${ }^{10}$ Department of Neonatology, Boai Hospital of Zhongshan, Zhongshan 528400, Guangdong, China. ${ }^{11}$ Department of Neonatology, Zhuhai Maternity and Child Health Hospital, Zhuhai 519001, Guangdong, China. ${ }^{12}$ Department of Neonatology, Huizhou Municipal Central Hospital, Huizhou 516001, Guangdong, China.

${ }^{13}$ Department of Neonatology, Dongguan Maternity and Child Health Hospital, Dongguan 523002, Guangdong, China. ${ }^{14}$ Department of Neonatology, Jiangmen Central Hospital, Affiliated Jiangmen Hospital of Sun Yat-sen University, Jiangmen 529000, Guangdong, China. ${ }^{15}$ Department of Neonatology, Yuebei People's Hospital, Shaoguan 512026, Guangdong, China. ${ }^{16}$ Department of Neonatology, Guangdong Second Provincial People's Hospital, Guangzhou 510317, Guangdong, China. ${ }^{17}$ Department of Pediatrics, Zhujiang Hospital of Southern Medical University, Guangzhou 510280, Guangdong, China. ${ }^{18}$ Department of Pediatrics, the Affiliated Hospital of 
Guangdong Medical University, Zhanjiang 524001, Guangdong, China. ${ }^{19}$ Department of Neonatology, the Second Affiliated Hospital of Shantou University Medical College, Shantou 515041, Guangdong, China. ${ }^{20}$ Department of Neonatology, Jinan University Medical College Affiliated Dongguan Hospital, Dongguan 523900, Guangdong, China. ${ }^{21}$ Department of Pediatrics, Dongguan People's Hospital, Dongguan 523000, Guangdong, China. ${ }^{22}$ Department of Neonatology, the First Affiliated Hospital of Sun Yat-sen University, Guangzhou 510080, Guangdong, China. ${ }^{23}$ Department of Neonatology, the First Affiliated Hospital of Shantou University Medical College, Shantou 515041, Guangdong, China. ${ }^{24}$ Department of Neonatology, Nanhai District People's Hospital of Foshan, Foshan 528200, Guangdong, China. ${ }^{25}$ Department of Neonatology, Huadu District People's Hospital of Guangzhou, Guangzhou 510800, Guangdong, China. ${ }^{26}$ Department of Pediatrics, the First People's Hospital of Zhaoqing, Zhaoqing 526020, Guangdong, China.

Received: 27 March 2019 Accepted: 20 September 2019 Published online: 04 November 2019

\section{References}

1. Doyle LW, Victorian Infant Collaborative Study Group. Evaluation of neonatal intensive care for extremely low birth weight infants in Victoria over two decades: I. effectiveness. Pediatrics. 2004;113(3 Pt 1):505-9.

2. Serenius F, Kallen K, Blennow M, Ewald U, Fellman V, Holmstrom G, Lindberg E, Lundqvist P, Marsal K, Norman M, et al. Neurodevelopmental outcome in extremely preterm infants at 2.5 years after active perinatal care in Sweden. JAMA. 2013;309(17):1810-20.

3. Stoll BJ, Hansen NI, Bell EF, Shankaran S, Laptook AR, Walsh MC, Hale EC, Newman NS, Schibler K, Carlo WA, et al. Neonatal outcomes of extremely preterm infants from the NICHD neonatal research network. Pediatrics. 2010;126(3):443-56.

4. de Waal CG, Weisglas-Kuperus N, van Goudoever JB, Walther FJ, NeoNed Study Group, LNF Study Group. Mortality, neonatal morbidity and two year follow-up of extremely preterm infants born in the Netherlands in 2007. PLoS One. 2012;7(7):e41302.

5. Costeloe KL, Hennessy EM, Haider S, Stacey F, Marlow N, Draper ES. Short term outcomes after extreme preterm birth in England: comparison of two birth cohorts in 1995 and 2006 (the EPICure studies). BMJ. 2012;345:e7976.

6. Shah PS, Sankaran K, Aziz K, Allen AC, Seshia M, Ohlsson A, Lee SK, Canadian Neonatal Network. Outcomes of preterm infants $<29$ weeks gestation over 10-year period in Canada: a cause for concern? J Perinatol. 2012;32(2):132-8.

7. Berger TM, Steurer MA, Woerner A, Meyer-Schiffer P, Adams M, Swiss Neonatal Network. Trends and Centre-to-Centre variability in survival rates of very preterm infants (<32 weeks) over a 10-year-period in Switzerland. Arch Dis Child Fetal Neonatal Ed. 2012;97(5):F323-8.

8. Doyle LW, Roberts G, Anderson PJ, Victorian Infant Collaborative Study Group. Outcomes at age 2 years of infants $<28$ weeks' gestational age born in Victoria in 2005. J Pediatr. 2010;156(1):49-53 e41.

9. Obstetrics Subgroup,Chinese Society of Obstetrics and Gynecology, Chinese Medical Association; Obstetrics Subgroup Chinese Society of Obstetrics and Gynecology Chinese Medical Association; Obstetrics Subgroup Chinese Society of Obstetrics and Gynecology Chinese Medical Association. Diagnosis and therapy guideline of preterm birth (2014). Zhonghua Fu Chan Ke Za Zhi. 2014;49(7):481-5.

10. Yang HX, Dong Y. Explanation on diagnosis and treatment guideline of preterm labor (protocol). Zhonghua Fu Chan Ke Za Zhi. 2007:42(7):433.

11. Bhakta K: Respiratory distress syndrome. . In: Manual of neonatal care. 7 Edited by JP. C. Philadelphia: Little, Brown and Company; 2012: 406-416.

12. Jobe AH, Bancalari E. Bronchopulmonary dysplasia. Am J Respir Crit Care Med. 2001;163(7):1723-9.

13. Bell MJ, Ternberg JL, Feigin RD, Keating JP, Marshall R, Barton L, Brotherton T. Neonatal necrotizing enterocolitis. Therapeutic decisions based upon clinical staging. Ann Surg. 1978;187(1):1-7.

14. International Committee for the Classification of Retinopathy of Prematurity. The international classification of retinopathy of prematurity revisited. Arch Ophthalmol. 2005;123(7):991-9.

15. Papile LA, Munsick-Bruno G, Schaefer A. Relationship of cerebral intraventricular hemorrhage and early childhood neurologic handicaps. J Pediatr. 1983;103(2):273-7.

16. Volpe JJ. Neurobiology of periventricular leukomalacia in the premature infant. Pediatr Res. 2001;50(5):553-62.
17. Ministry of Health of the People's Republic of China: Notice about diagnostic criteria of hospital infection. http://www.nhc.gov.cn/wjw/gfxwj/2 01304/37cad8d95582456d8907ad04a5f3bd4c.shtml. Accessed 3 Aug 2019.

18. World Health Organization. March of Dimes; The Partnership for Maternal, Newborn \& Child Health; Save the Children: Born too soon: the global action report on preterm birth. http://www.who.int/maternal_child_ adolescent/documents/born_too_soon/en/. Accessed 3 Aug 2019.

19. Blencowe $H$, Cousens S, Oestergaard MZ, Chou D, Moller AB, Narwal R, Adler A, Vera Garcia C, Rohde S, Say L, et al. National, regional, and worldwide estimates of preterm birth rates in the year 2010 with time trends since 1990 for selected countries: a systematic analysis and implications. Lancet. 2012;379(9832):2162-72.

20. Isayama T, Lee SK, Mori R, Kusuda S, Fujimura M, Ye XY, Shah PS, Canadian Neonatal Network, Neonatal Research Network of Japan. Comparison of mortality and morbidity of very low birth weight infants between Canada and Japan. Pediatrics. 2012;130(4):e957-65.

21. Ma L, Liu C, Cheah I, Yeo KT, Chambers GM, Kamar AA, Travadi J, Oei JL. Cost is an important factor influencing active management of extremely preterm infants. Acta Paediatr. 2019;108(1):70-5.

22. Lundqvist $P$, Kallen $K$, Hallstrom I, Westas LH. Trends in outcomes for very preterm infants in the southern region of Sweden over a 10-year period. Acta Paediatr. 2009;98(4):648-53.

23. Schmidt B, Asztalos EV, Roberts RS, Robertson CM, Sauve RS, Whitfield MF, Trial of Indomethacin Prophylaxis in Preterms I. Impact of

bronchopulmonary dysplasia, brain injury, and severe retinopathy on the outcome of extremely low-birth-weight infants at 18 months: results from the trial of indomethacin prophylaxis in preterms. JAMA. 2003;289(9):1124-9.

24. Bassler D, Stoll BJ, Schmidt B, Asztalos EV, Roberts RS, Robertson CM, Sauve RS, Trial of Indomethacin Prophylaxis in Preterms I. Using a count of neonatal morbidities to predict poor outcome in extremely low birth weight infants: added role of neonatal infection. Pediatrics. 2009;123(1):313-8.

25. Gargus RA, Vohr BR, Tyson JE, High P, Higgins RD, Wrage LA, Poole K. Unimpaired outcomes for extremely low birth weight infants at 18 to 22 months. Pediatrics. 2009;124(1):112-21.

26. Roberts D, Brown J, Medley N, Dalziel SR. Antenatal corticosteroids for accelerating fetal lung maturation for women at risk of preterm birth. Cochrane Database Syst Rev. 2017;3:CD004454.

27. Bolisetty S, Legge N, Bajuk B, Lui K, New South W, the Australian Capital Territory Neonatal Intensive Care Units' Data Collection. Preterm infant outcomes in New South Wales and the Australian Capital Territory. J Paediatr Child Health. 2015;51(7):713-21.

28. Stoll BJ, Hansen NI, Bell EF, Walsh MC, Carlo WA, Shankaran S, Laptook AR, Sanchez PJ, Van Meurs KP, Wyckoff M, et al. Trends in care practices, morbidity, and mortality of extremely preterm neonates, 1993-2012. JAMA. 2015;314(10):1039-51.

29. Kattwinkel J, Perlman JM, Aziz K, Colby C, Fairchild K, Gallagher J, Hazinski MF, Halamek LP, Kumar P, Little G, et al. Part 15: neonatal resuscitation: 2010 American Heart Association guidelines for cardiopulmonary resuscitation and emergency cardiovascular care. Circulation. 2010;122(18 Suppl 3):S909-19.

\section{Publisher's Note}

Springer Nature remains neutral with regard to jurisdictional claims in published maps and institutional affiliations.

\section{Ready to submit your research? Choose BMC and benefit from:}

- fast, convenient online submission

- thorough peer review by experienced researchers in your field

- rapid publication on acceptance

- support for research data, including large and complex data types

- gold Open Access which fosters wider collaboration and increased citations

- maximum visibility for your research: over $100 \mathrm{M}$ website views per year

At BMC, research is always in progress.

Learn more biomedcentral.com/submissions 